\title{
Screening Status as a Determinant of Choice of Colorectal Cancer Screening Method: A Population-Based Informed Survey
}

\author{
José Luis Sandoval ${ }^{a, b}$ Allan Relecom ${ }^{b}$ Cyril Ducros $^{c}$ Jean-Luc Bulliard ${ }^{d}$ \\ Beatrice Arzel ${ }^{\mathrm{e}}$ Idris Guessous ${ }^{\mathrm{a}}$ \\ aUnit of Population Epidemiology, Division of Primary Care Medicine, Department of Primary Care Medicine, \\ Geneva University Hospitals, Geneva, Switzerland; 'bepartment of Oncology, Geneva University Hospitals, Geneva, \\ Switzerland; 'Vaud Cancer Screening Foundation, Lausanne, Switzerland; 'Institute of Social and Preventive \\ Medicine, University Hospital of Lausanne, Lausanne, Switzerland; 'Geneva Cancer Screening Foundation, Geneva, \\ Switzerland
}

\section{Keywords \\ Colon cancer · Screening · Fecal blood testing · \\ Colonoscopy $\cdot$ Patient choice}

\begin{abstract}
Objectives: Fecal blood testing is a noninvasive alternative to colonoscopy for colorectal cancer (CRC) screening and is preferred by a substantial proportion of individuals. However, participant-related determinants of the choice of screening method, particularly up-to-date screening status, remain less studied. We aimed to determine if up-to-date screening status was related to choosing a fecal blood test over colonoscopy. Setting: Participants in the populationbased cross-sectional survey study Bus Santé in Geneva, Switzerland - aged 50-69 years. Design: Cross-sectional survey study using mailed questionnaires inquiring about CRC screening method of choice after providing information on advantages and disadvantages of both screening methods. We used multivariable logistic regression models to determine the association between up-to-date CRC screening status and choosing fecal blood testing. Key results: We included 1,227 participants. Thirty-eight percent of participants did not have up-to-date CRC screening. Overall, colonosco-
\end{abstract}

karger@karger.com www.karger.com/gat

Karger $\frac{1}{\%}$

GOPEN ACCESS
(C) 2021 The Author(s)

Published by S. Karger AG, Basel

This article is licensed under the Creative Commons AttributionNonCommercial-NoDerivatives 4.0 International License (CC BY NC-ND) (http://www.karger.com/Services/OpenAccessLicense) Usage and distribution for commercial purposes as well as any distribution of modified material requires written permission. py $(54.9 \%)$ was preferred to fecal blood testing $(45.1 \%)(p<$ 0.001 ) as screening method of choice. However, screening method choices differed between those with $(65.6 \%$ colonoscopy and $34.4 \%$ fecal blood testing) and without up-todate CRC screening (36.5\% colonoscopy and $63.5 \%$ fecal blood testing). Not having up-to-date CRC screening was associated with a higher probability of choosing fecal blood testing as screening method (odds ratio $=2.6[1.9 ; 3.7], p<$ 0.001 ) after adjustment for the aforementioned confounders. Conclusions: Not having up-to-date screening was independently associated with fecal blood testing as the preferred method for CRC screening. Proposing this method to this subpopulation, in a context of shared decision, could potentially increase screening uptake in settings where it is already high.

(c) 2021 The Author(s).

Published by S. Karger AG, Basel

\section{Introduction}

Colorectal cancer (CRC) is the third most common cancer worldwide, accounting for 832,000 deaths and a burden of 17 million disease-adjusted life years in 2015 [1]. Furthermore, its yearly incidence has increased $37 \%$ 
from 2005 to 2015 , from 1.2 to 1.7 million new cases, reflecting the overall population growth and aging [1].

Early detection is a key determinant of survival, with the development of screening methods and strategies playing a fundamental role in decreasing the mortality and morbidity burden of CRC [2-4]. CRC screening participation has increased over time with, for instance, an increase from $33.9 \%$ in 2000 to $62.4 \%$ in 2015 in the USA [5].

One of the main recommended methods for CRC screening consists of a colonoscopy exam every 10 years [6]. However, due to the disadvantages of the method, such as physical discomfort, the potential need for sedation and high costs, screening uptake is variable and has remained low or suboptimal [7]. Noninvasive methods allow CRC screening by detecting occult fecal blood, but require more frequent screening (1-2 years) and a diagnostic colonoscopy in case of a positive result [3, 8-11].

Two randomized control trials in Spain and the USA are ongoing to detect differences in 10-year CRC mortality between patients screened either with fecal immunochemical test (FIT) (an occult fecal blood test) or colonoscopy $[10,12]$. Interim results from the Spanish trial revealed a similar detection rate of CRC with either method and, however, a higher adenoma detection rate was reported with colonoscopy [10]. A recent review on CRC screening by an expert working group under the auspices of the International Agency for Research on Cancer concluded that the 2 approaches effectively reduce the risk of death by CRC with their benefits outweighing the potential harms [6].

Thus, the choice of which method to use should consider patients' preferences and values, in a context of shared decision-making, with a potential to increase CRC screening uptake $[13,14]$. As such, understanding screening method preferences in a given population is fundamental when organized CRC screening programs are planned to ensure maximum uptake. Such a program is planned to be implemented in the State of Geneva, Switzerland, during 2019. Briefly, inhabitants aged 50-69 years will be mailed invitations to schedule an appointment with an affiliated doctor to choose between a FIT every 2 years and a colonoscopy every 10 years. The invitations will be sent together with decision aids detailing the advantages and disadvantages of each method. The appointment with the doctor and the screening procedure, independently of the chosen method, will be subsidized, with $10 \%$ of the costs being charged to the participant.

Several nonmodifiable (e.g., income, education, and age) and modifiable factors (e.g., structural barriers and previous knowledge about screening and CRC) were identified as determinants of screening participation [15, 16]. While the characteristics of each screening method that are more appealing to individuals have been identified $[17,18]$, participant-related determinants of screening method of choice are less studied.

It remains unclear whether participants' choices of screening method are dependent on their screening status independently of sociodemographic characteristics, family history of CRC, or access to medical advice. Determining which subpopulations would be more amenable to each screening modality could help doctors discuss the choice of screening method and contribute to increasing uptake. Thus, we aimed to determine if not having up-todate screening (screening status) is independently associated with the choice of CRC screening method after individuals are clearly informed of their advantages and limitations.

\section{Methods}

\section{Participants}

We used data from the Bus Santé, a population-based study ongoing since 1992 in the State of Geneva (approximately 500,000 people in 2016), monitoring population health and risk factors. Sampling strategy and data recording were described elsewhere [19]. Approximately 6,000 participants in the Bus Santé study with ages between 50 and 69 years have consented to be contacted to participate in further health-related studies. We randomly selected 3,000 to be mailed questionnaires concerning CRC screening. A reminder was sent if no response was obtained 2 months after the first contact (1,800 reminders). The questionnaire was first sent in June 2016 and the reminder in August 2016.

We obtained 1,310 (43.7\%) complete questionnaires. Approximately $4 \%(n=50)$ of the questionnaires were not interpretable (e.g., with duplicated identifiers) and were excluded from the final dataset $(n=1,260)$. The median time between participation in the Bus Santé Study and in this specific survey was 5.9 years (interquartile range $=4.3-7.2$ ). Written consent was obtained from all participants and this study received approval from the Institute of Ethics Committee of the University of Geneva.

\section{Questionnaire}

A first section of the questionnaire recorded data on personal and family history of CRC and if screening had been discussed with a doctor. A second section inquired about past use of colonoscopy/fecal blood CRC screening and when it was last performed. A third section evaluated the opinions concerning the introduction of an organized screening program. A fourth section contained graphic decision aids on the impact of CRC screening on the population and informed in a lay language about the pros and cons of colonoscopy and fecal immunological testing. The decision aids were developed for the Vaud CRC screening program in Switzerland after a review of the literature and revised by experts in cancer screening (https://bit.ly/2MQRcdv) [20]. The question-
Sandoval/Relecom/Ducros/Bulliard/ Arzel/Guessous 
naire was developed and tested by the collaborators of the Unit of Population Epidemiology and the Geneva Foundation for Cancer Screening.

\section{Variables}

Nationality was defined as either Swiss or not Swiss, and educational attainment was divided into 3 categories as in Huissman et al. [21]: (1) no end of school certification (Maturité) or no professional apprenticeship (primary); (2) obtaining "Maturité" or professional apprenticeship (secondary); and (3) university degree (tertiary). Monthly household income was self-reported in CHF $(<2,999,3,000-4,999,5,000-6,999,7,000-9,499,9,500-13,000$, or $>13,000)$ in the questionnaire and adjusted for household size using the ratio: income/(household size) ${ }^{0.5}$ [22]. In 2016, 1 CHF corresponded to approximately 1 USD. Previous discussion with a doctor (yes or no), family history (yes or no), and personal history (yes or no) of CRC, as well as occupation (employed and nonmanual, employed and manual, executive or independent, and nonmanual, invalidity retirement, retired, unemployed, studying, or housewife/househusband), were collected in the questionnaire.

Up-to-date screening by colonoscopy was defined as having had a colonoscopy in the 10 years before the survey. For fecal blood testing, up-to-date screening was considered when the test had been performed within 2 years of being surveyed. Overall up-todate screening was classified as yes if screening was up-to-date using any of the 2 methods. The outcome variable - choice of screening method - was obtained from the questionnaire using the question "based on the preceding information, which method would you tend to choose in the first place for colorectal cancer screening?" which followed the explanation on the impact of screening as well as advantages and disadvantages of fecal immunological testing and colonoscopy.

In addition, participants were also asked to identify how several factors influenced (no influence, some influence, or a lot of influence) the choice of screening method. These factors included differences in screening frequency, monetary cost, practical disadvantages of colonoscopy (i.e., need for preparation with a laxative the day before, travel costs to the gastroenterologist's office, impossibility to drive a vehicle after the procedure if sedation was needed), practical disadvantages of fecal blood testing (i.e., collecting the kit from a pharmacist, handling own stool), colonoscopy complication risks, better detection of polyps by colonoscopy, and the need of colonoscopy if positive fecal blood test. All information regarding these factors was included in the description of the 2 methods that preceded this section of the questionnaire, and examples were repeated at each question to ensure their clarity.

\section{Statistical Analysis}

Categorical variables are presented as absolute and relative frequencies and continuous ones as median and interquartile range. We used Wilcoxon rank-sum and $\chi^{2}$ test of independence to determine the significance of differences between groups for continuous and categorical variables, respectively. We excluded individuals with a personal history of CRC $(n=17,1.4 \%)$ and those older than 69 years $(n=16,1.3 \%)$ as these subjects will be ineligible for the Geneva CRC screening program.

We converted the nominal rating scale used by the participants to grade the factors that influenced their choice into a numerical equivalent (0-no influence, 1 -some influence, and 2-a lot of influence). Average scores for each factor were calculated for the 2 groups defined according to screening status. These were compared using $T$ tests for independent samples, despite the nonparametric nature of the data, as previously suggested [23].

A multivariable logistic regression analysis was performed to determine the influence of screening status on the choice of screening method, adjusting for demographic variables (age, nationality, and gender), family history of CRC, previous discussion about screening with a doctor, and socioeconomic status indicators (educational attainment and occupational level). We further analyzed the effect of adjusted household income in a subset of participants for which this information was available. Due to substantial missing data for adjusted household income ( $n=487,39.7 \%)$, we report this subanalysis in the online suppl. Material; for all online suppl. material, see www.karger.com/doi/10.1159/000512954 (online suppl. Table 2). Crude and adjusted odds ratios (ORs) are reported with $95 \%$ confidence intervals (CIs). A two-sided $p<0.05$ was considered significant and data were analyzed using STATA 15.1 and R 3.2.2.

\section{Results}

\section{Participants' Characteristics}

Participants' characteristics are presented in Table 1. Median age of participants was 58.1 years, $52.3 \%$ were female, 76.4\% Swiss nationals, 70.2\% had already discussed CRC screening with their doctors, and $17.8 \%$ had a family history of CRC. Median adjusted household income was 5,834 USD, and $46.6 \%$ had tertiary education. The majority were employed with a nonmanual occupation $(26.7 \%)$, retired $(26.0 \%)$ or executives or independent with a nonmanual occupation $(22.0 \%)$.

Regarding screening, $62.0 \%$ were up-to-date, $7.4 \%$ using fecal blood testing, and $59.0 \%$ by colonoscopy. Colonoscopy $(54.9 \%)$ was preferred to fecal blood testing $(45.1 \%)(p<0.001)$ as screening method of choice.

Participants without up-to-date CRC screening were younger, more often female, had less often a family history of CRC, had less often been informed about screening by a doctor, and were less often retired than participants with up-to-date CRC screening status (Table 1). Furthermore, $11 \%$ of those without up-to-date screening had already had a colonoscopy in the past (i.e., $>10$ years ago). Participants with up-to-date screening had it, in most cases, using colonoscopy (96.3\%), while $12.7 \%$ had up-to-date fecal blood testing. Household income and educational attainment did not differ between the 2 groups (Table 1). Those with up-to-date screening preferred colonoscopy (65.6\%) over fecal blood testing (34.4\%) whereas those without up-to-date screening preferred fecal blood testing $(63.5 \%)$ over colonoscopy $(36.5 \%)(p<0.001)$. 
Table 1. Participants' characteristics

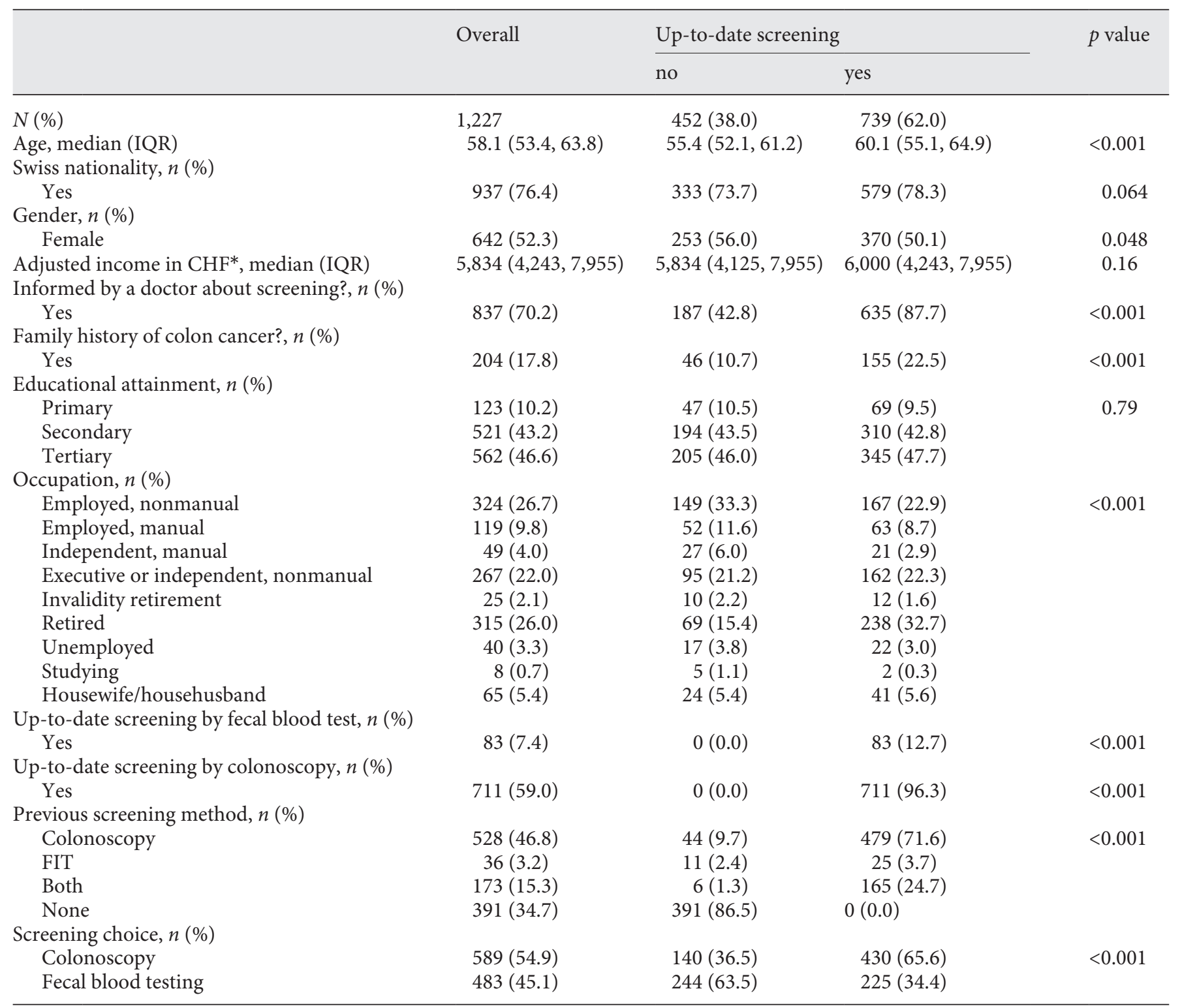

IQR, interquartile range; FIT, fecal immunochemical test. * 1 USD, 1 CHF in 2016.

Participants' characteristics according to method of choice and method used in previous screening, if any, can be found in online suppl. Tables 1 and 4, respectively. Those preferring colonoscopy were more often male, had previously discussed screening with their doctors, and had a more often a family history of colon cancer (online suppl. Table 1). The participants that had a colonoscopy in the past, compared to those that only had FIT, were older, more often had a family history of colon cancer and preferred colonoscopy over FIT as future screening choice (online suppl. Table 4).

\section{Reasons for Choice of Method}

The analysis of the features that influenced the choice of preferred screening method (Fig. 1) revealed significant differences between the 2 groups in 4 items: higher costs of colonoscopy, colonoscopy's practical disadvantages, risk of colonoscopy complications, and better de-
66

Gastrointest Tumors 2021;8:63-70 DOI: $10.1159 / 000512954$
Sandoval/Relecom/Ducros/Bulliard/ Arzel/Guessous 


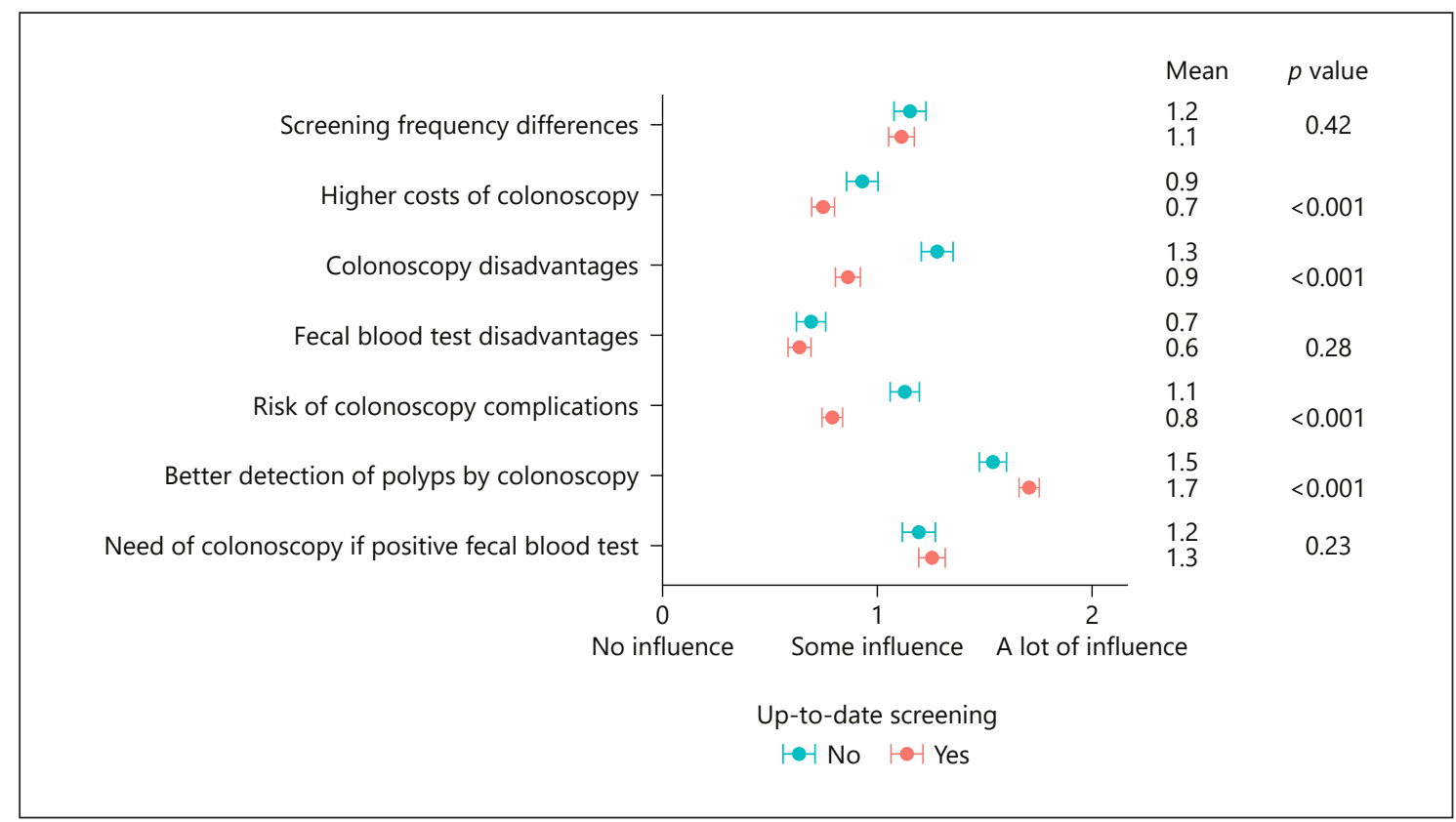

Fig. 1. Factors influencing screening method choice among participants with (red) and without (blue) up-to-date CRC screening. Mean and $95 \%$ CIs are presented based on a rating scale ( 0 - no influence, 1 - some influence, 2 - a lot of influence). Independent samples Student's $T$ test $p$ values are presented comparing the 2 groups. CRC, colorectal cancer; CI, confidence interval.

tection of polyps using colonoscopy. The first 3 items were found to be more influential for those without upto-date screening (colonoscopy costs $0.795 \% \mathrm{CI}=[0.7$; $0.8]$ vs. 0.9 [0.9; 1.0], disadvantages of colonoscopy 0.9 $[0.8 ; 0.9]$ vs. $1.3[1.2 ; 1.4]$, and colonoscopy's complications $0.8[0.7 ; 0.8]$ vs. $1.1[1.1 ; 1.2])$, while the better detection of polyps by colonoscopy was more influential for those with up-to-date screening $(1.7[1.7 ; 1.8]$ vs. $1.5[1.5$; 1.6]).

\section{Association between Screening Status and Choice of Screening Method}

Participants without up-to-date screening had an increased probability of choosing a fecal blood test rather than a colonoscopy $(\mathrm{OR}=3.395 \% \mathrm{CI}=[2.6 ; 4.3])(\mathrm{Ta}-$ ble 2). The effect of screening status on screening method of choice was independent of gender and nationality $(\mathrm{OR}=3.4[2.6 ; 4.5])$, family history of CRC and discussion with a doctor concerning screening $(\mathrm{OR}=2.6[1.9$; 3.6]), and educational and occupational level $(\mathrm{OR}=2.6$ $[1.9 ; 3.7]$ ) (Table 2). Even after adjustment for income $(\mathrm{OR}=2.3[1.5 ; 3.4])$, screening status remained an independent predictor of CRC screening method of choice (online suppl. Table 2). Past method of screening was associated with method choice for future screening. Participants who had screening using exclusively FIT were more likely to prefer this method to colonoscopy than those previously screened using colonoscopy exclusively $\left(\mathrm{OR}_{\text {FIT vs. colonoscopy }}=9.6[3.9 ; 26.7]\right)$. Nevertheless, screening status was still associated with method of choice for future screening when the analysis was adjusted for past screening habits $(\mathrm{OR}=2.6[1.3 ; 5.2]$, online suppl. Table 5).

Furthermore, socioeconomic status indicators were not associated with the choice of screening method $(p>$ 0.05 ) (Table 2). Interestingly, having had a previous discussion with a doctor concerning CRC screening was associated with decreased probability of choosing fecal blood testing over colonoscopy $(\mathrm{OR}=0.4[0.3 ; 0.6])(\mathrm{Ta}-$ ble 2).

\section{Discussion}

Our results show that individuals without up-to-date CRC screening prefer fecal blood testing over colonoscopy. Moreover, this choice pattern was independent of multiple individual characteristics related to demograph- 
Table 2. Unadjusted and adjusted logistic regression models for the association between up-to-date screening status and choosing fecal blood test as screening method

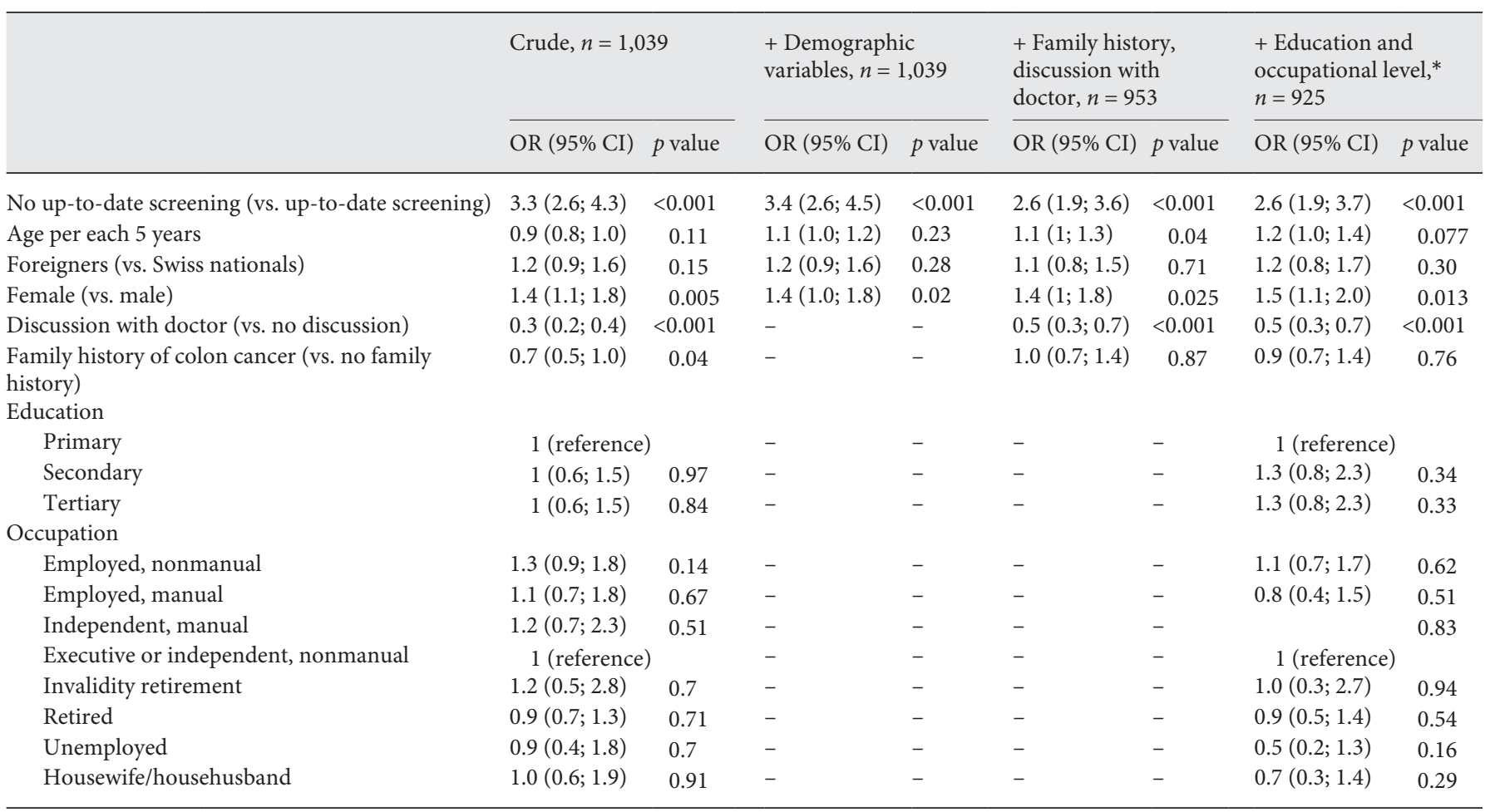

OR, odds ratio; CI, confidence interval. * Students were removed due to low number of participants in this category.

ics, socioeconomic status, family history of CRC, previous discussion with a doctor concerning screening, and previous method of screening.

Currently, various CRC screening methods are equally recommended by several guidelines, including those of the US Preventive Services Task Force [24]. However, identifying the methods that increase uptake and in which individuals has been a challenge. In a study of colonoscopy naive adults, DeBourcy et al. [25] found a preference for fecal occult blood testing over colonoscopy, but the overall preferences of the population (including those that were not colonoscopy naive) were not determined.

In France, Rat et al. [26] found that providing general practitioners with reminders containing lists of their patients who had not undergone CRC screening led to an increase in screening by FIT. In California, Singal et al. [27] observed that mailed FIT outreach resulted in a higher CRC screening uptake compared to no outreach or mailed colonoscopy outreach.

Our results suggest that individuals without up-todate screening may be more susceptible to suggestions to undergo fecal blood testing rather than colonoscopy, possibly increasing screening uptake. On the other hand, those with up-to-date screening are more likely to continue with their current choice (in the case of our study, colonoscopy).

In our study, discussing with a doctor about CRC screening was associated with a lower probability of choosing fecal blood testing. This could mean that doctors are reassuring individuals on the favorable benefitrisk ratio of undergoing a colonoscopy or that doctors are mainly suggesting screening by colonoscopy. This latter assumption is confirmed by the results of a recent survey of CRC screening practices and preferences among general practitioners in the state of Geneva which indicate a strong tendency to practice and recommend colonoscopy over fecal occult blood test [28]. The discrepancy between patient and doctor's screening method of choice has been previously observed [18]. Moreover for both doctors and patients, the choice is driven by which method features they value the most. Those who valued accuracy tended to select colonoscopy, while those who valued inconve-
68

Gastrointest Tumors 2021;8:63-70 DOI: $10.1159 / 000512954$
Sandoval/Relecom/Ducros/Bulliard/ Arzel/Guessous 
nience, complications and discomfort chose a fecal blood testing method [18].

Similarly, we observed differences in the features driving screening method of choice between those with and without up-to-date screening. Participants without upto-date screening were more concerned about the risks of colonoscopy, its costs, and potential complications. Individuals with up-to-date screening were more concerned about the accuracy of colonoscopy in detecting a higher number of polyps.

While we did not compare the doctors' method of choice to those of their patients, other studies have reported that increasing shared decision could align patients' and doctors' opinions on the method of choice with a potential increase in screening uptake $[13,14]$. As such, we could argue that, in a context of shared decision, suggesting FIT to patients that do not have up-to-date screening or not favoring colonoscopy as the main doctor recommendation could have a positive impact in overall screening uptake. This may be particularly important in contexts where uptake is already high, as suggested by our results for Geneva, where the main barriers for screening participation may be related to the screening procedure rather than lack of information concerning its importance. However, further studies, including intervention studies, are needed to validate this hypothesis.

\section{Strengths}

To the best of our knowledge, this is the first study addressing the importance of screening status on screening method of choice after participants were presented with the advantages and disadvantages of the 2 main CRC screening methods. We used data from an ongoing population-based cross-sectional study allowing us to capture data for a wide range of sociodemographic indicators and determine if the choice was socially patterned. In particular, we were able to assess any difference in characteristics between participants and nonparticipants (see below). Through including questions regarding the motivations behind the choice, we were able to uncover screening method features that were valued by different groups according to screening status and that may lead to low screening uptake if the wrong options are presented as the preferred method of choice by the doctor.

\section{Limitations}

Our study has several limitations. First, and similar to other survey studies, the response rate was moderate (43.7\%). We compared the descriptives between partici- pants and nonparticipants and the former were more often Swiss (76.6 vs. $66.2 \%)$ and had more often tertiary education ( 46.7 vs. $37.5 \%$ ) than the latter (online suppl. Table 3). While the differences between participants and nonparticipants were moderate, our study participants may represent the adults who would actually participate in the future organized screening program, being more prone to answering a mail invitation to participate. Second, this study was based on a single region (the State of Geneva). Replication is needed in other Swiss regions and other countries to assess the generalizability of our findings. Third, while we used various sociodemographic indicators and variables related to family history and previous discussion with a doctor concerning CRC screening, additional confounding by unmeasured factors and social desirability bias, inherent to any self-reported data, cannot be excluded.

\section{Conclusions}

In a population with a relatively high CRC screening uptake, we identified not having up-to-date screening as being independently associated with choosing a fecal blood analysis-based over colonoscopy. This preference was observed after clear and balanced information about the advantages and disadvantages of each method was presented.

Our findings could help inform clinicians and screening programs to tailor the suggested methods to fit patients' preferences. Favoring shared decision-making and a better concordance between patients' preferences and what is suggested to them may increase screening uptake and, consequently, improve its impact on reducing the global burden of disease attributed to CRC.

\section{Acknowledgements}

The authors would like to acknowledge all the Bus Santé participants that kindly contributed with their data. We thank Jean Marc Theler for his contribution to the data management of the Bus Santé dataset.

\section{Statement of Ethics}

This study complies with the ethical standards established by the Declaration of Helsinki. Informed consent was obtained from all participants. It was approved by the Institute of Ethics Committee of the University of Geneva. 


\section{Conflict of Interest Statement}

The authors have no conflicts of interest to declare.

\section{Funding Sources}

The Bus Santé Study is funded by the Geneva University Hospitals and the General Directorate of Health, Canton of Geneva, Switzerland.

\section{Author Contributions}

José Luis Sandoval: conceptualization, analysis and interpretation of results, and manuscript writing and revision. Allan Relecom, Cyril Ducros, Jean-Luc Buillard, Beatrice Arzel: data collection, interpretation of results, manuscript reviewing, and final editing the final manuscript. Idris Guessous: conceptualization, data collection, interpretation of results, and manuscript writing and revision.

\section{References}

1 Fitzmaurice C, Allen C, Barber RM, Barregard L, Bhutta ZA, Brenner H, et al. Global, regional, and national cancer incidence, mortality, years of life lost, years lived with disability, and disability-adjusted life-years for 32 cancer groups, 1990 to 2015: a systematic analysis for the global burden of disease study. JAMA Oncol. 2017;3(4):524-48.

2 Holme $\varnothing$, Bretthauer M, Fretheim A, Odgaard-Jensen J, Hoff G. Flexible sigmoidoscopy versus faecal occult blood testing for colorectal cancer screening in asymptomatic individuals. Cochrane Database Syst Rev. 2013(9):CD009259...

3 Mandel JS, Bond JH, Church TR, Snover DC, Bradley GM, Schuman LM, et al. Reducing mortality from colorectal cancer by screening for fecal occult blood. Minnesota colon cancer control study. N Engl J Med. 1993;328: 1365-71.

4 Selby JV, Friedman GD, Quesenberry CP Jr, Weiss NS. A case-control study of screening sigmoidoscopy and mortality from colorectal cancer. N Engl J Med. 1992;326(10):653-7.

5 Health, United States, 2016: with chartbook on long-term trends in health. Hyattsville, MD: National Center for Health Statistics 2017.

6 Lauby-Secretan B, Vilahur N, Bianchini F, Guha N, Straif K. The IARC perspective on colorectal cancer screening. N Engl J Med. 2018;378(18):1734-40.

7 Navarro M, Nicolas A, Ferrandez A, Lanas A. Colorectal cancer population screening programs worldwide in 2016: an update. World J Gastroenterol. 2017;23(20):3632-42.

8 Hardcastle JD, Thomas WM, Chamberlain J, Pye G, Sheffield J, James PD, et al. Randomised, controlled trial of faecal occult blood screening for colorectal cancer. Results for first 107,349 subjects. Lancet. 1989; 1(8648):1160-4.

9 Mandel JS, Church TR, Ederer F, Bond JH. Colorectal cancer mortality: effectiveness of biennial screening for fecal occult blood. I Natl Cancer Inst. 1999;91(5):434-7.

10 Quintero E, Castells A, Bujanda L, Cubiella J, Salas D, Lanas Á, et al. Colonoscopy versus fecal immunochemical testing in colorectalcancer screening. N Engl J Med. 2012;366(8): 697-706.
11 Smith A, Young GP, Cole SR, Bampton P. Comparison of a brush-sampling fecal immunochemical test for hemoglobin with a sensitive guaiac-based fecal occult blood test in detection of colorectal neoplasia. Cancer. 2006; 107(9):2152-9.

12 Dominitz JA, Robertson DJ, Ahnen DJ, Allison JE, Antonelli M, Boardman KD, et al. Colonoscopy vs. fecal immunochemical test in reducing mortality from colorectal cancer (CONFIRM): rationale for study design. Am J Gastroenterol. 2017;112(11):1736-46.

13 Briss P, Rimer B, Reilley B, Coates RC, Lee NC, Mullen P, et al. Promoting informed decisions about cancer screening in communities and healthcare systems. Am J Prev Med. 2004;26(1):67-80.

14 Schroy PC 3rd, Emmons KM, Peters E, Glick JT, Robinson PA, Lydotes MA, et al. Aid-assisted decision making and colorectal cancer screening: a randomized controlled trial. Am J Prev Med. 2012;43(6):573-83.

15 Gimeno Garcia AZ. Factors influencing colorectal cancer screening participation. Gastroenterol Res Pract. 2012;2012:483417.

16 Guessous I, Dash C, Lapin P, Doroshenk M, Smith RA, Klabunde CN. Colorectal cancer screening barriers and facilitators in older persons. Prev Med. 2010;50(1-2):3-10.

17 Hol L, de Bekker-Grob EW, van Dam L, Donkers B, Kuipers EJ, Habbema JD, et al. Preferences for colorectal cancer screening strategies: a discrete choice experiment. $\mathrm{Br} \mathrm{J}$ Cancer. 2010;102(6):972-80.

18 Ling BS, Moskowitz MA, Wachs D, Pearson B, Schroy PC. Attitudes toward colorectal cancer screening tests. J Gen Intern Med. 2001;16(12):822-30.

19 Guessous I, Bochud M, Theler JM, Gaspoz JM, Pechère-Bertschi A. 1999-2009 trends in prevalence, unawareness, treatment and control of hypertension in Geneva, Switzerland. PLoS One. 2012;7(6):e39877.

20 Selby K, Cornuz J, Gachoud D, Bulliard JL, Nichita C, Dorta G, et al. Training primary care physicians to offer their patients faecal occult blood testing and colonoscopy for colorectal cancer screening on an equal basis: a pilot intervention with before-after and parallel group surveys. BMJ Open. 2016;6(5): e011086.
21 Huisman M, Kunst AE, Mackenbach JP. Educational inequalities in smoking among men and women aged 16 years and older in $11 \mathrm{Eu}$ ropean countries. Tob Control. 2005;14(2): 106-13.

22 Garner TI, Ruiz-Castillo J, Sastre M. The influence of demographics and household-specific price indices on consumption-based inequality and welfare: a comparison of spain and the United States. South Econ J. 2003;70: 22-48.

23 Sullivan GM, Artino AR Jr. Analyzing and interpreting data from likert-type scales. J Grad Med Educ. 2013;5(4):541-2.

24 Bibbins-Domingo K, Bibbins-Domingo K, Grossman DC, Curry SJ, Davidson KW Jr, Epling JW, et al. Screening for colorectal cancer: US preventive services task force recommendation statement. JAMA. 2016;315(23): 2564-75.

25 DeBourcy AC, Lichtenberger S, Felton S, Butterfield KT, Ahnen DJ, Denberg TD. Community-based preferences for stool cards versus colonoscopy in colorectal cancer screening. J Gen Intern Med. 2008;23(2):169-74.

26 Rat C, Pogu C, Le Donné D, Latour C, Bianco G, Nanin F, et al. Effect of physician notification regarding nonadherence to colorectal cancer screening on patient participation in fecal immunochemical test cancer screening: a randomized clinical trial. JAMA. 2017; 318(9):816-24

27 Singal AG, Gupta S, Skinner CS, Ahn C, Santini NO, Agrawal D, et al. Effect of colonoscopy outreach vs. fecal immunochemical test outreach on colorectal cancer screening completion: a randomized clinical trial. JAMA. 2017;318(9):806-15.

28 Bulliard JL, Germann S. Enquête sur le dépistage du cancer colorectal auprès des médecins de premier recours genevois. Lausanne, Division des maladies chroniques, Institut universitaire de médecine sociale et préventive (IUMSP). 2016. 